\title{
Selection for resistance to a fungal pathogen in Drosophila melanogaster
}

\author{
AR Kraaijeveld ${ }^{1}$ and HCJ Godfray ${ }^{2}$ \\ NERC Centre for Population Biology, Imperial College London, Silwood Park Campus, Ascot, Berks, UK
}

An artificial selection experiment designed to explore the evolution of resistance to a fungal pathogen, Beauveria bassiana, in Drosophila melanogaster is reported here. The experiment was designed to test whether there is sufficient additive genetic variation in this trait for increased resistance to evolve, and, if so, whether there are correlated responses that might represent a cost to defence. After 15 generations of selection, flies from selected lines did not have higher overall fitness after infection compared with control lines. The response to selection for resistance against this pathogen is thus much weaker than against other species, in particular, parasitoids. There was, however, evidence for increased late-life fecundity in selected lines, which may indicate evolved tolerance of fungal infection. This increase was accompanied by reduced early-life fitness, which may reflect the well-known trade-off between early and late reproduction. In the absence of fungal infection, selected flies had lower fitness than control flies, and the possibility that this is also a trade-off with increased tolerance is explored.

Heredity (2008) 100, 400-406; doi:10.1038/sj.hdy.6801092; published online 27 February 2008

Keywords: artificial selection; Beauveria bassiana; Drosophila melanogaster, fungal pathogen; immunity; fungal resistance

\section{Introduction}

All organisms need to defend themselves against pathogens, parasites and other natural enemies, and investment in defence is a significant component of the life history strategy of most animals and plants (Sheldon and Verhulst, 1996; Schmid-Hempel and Ebert, 2003). The extent to which investment in defence is favoured by natural selection depends on the probability of attack, the likelihood that resistance will be successful and the costs of redirecting resources to defence from other life history functions that influence fitness (Rolff and SivaJothy, 2003). In addition, the evolution of resistance requires additive genetic variation in this trait. Assessing the costs of defence is not easy, as correlative studies are hard to interpret due to confounding variables, while direct experimental manipulation is difficult because most resistance mechanisms involve complex physiological responses. An attractive alternative is to use artificial selection to increase resistance to a natural enemy and then look for correlated responses to selection that might represent costs. Here, we report an investigation, using artificial selection, into the ability of Drosophila melanogaster to evolve resistance to the fungal pathogen Beauveria bassiana, and whether there are costs to improved defence.

Correspondence: $\operatorname{Dr}$ AR Kraaijeveld, School of Biological Sciences, University of Southampton, Bassett Crescent East, Southampton, Hants SO16 7PX, UK

E-mail: arkraa@soton.ac.uk

${ }^{1}$ Current address: School of Biological Sciences, University of Southampton, Southampton, Hants, UK.

${ }^{2}$ Current address: Department of Zoology, University of Oxford, Oxford, UK.

Received 3 September 2007; revised 9 November 2007; accepted 11 December 2007; published online 27 February 2008
In addition to studies on Drosophila that we discuss in more detail below, artificial selection has been used to identify costs of resistance in several other insects, for example, in the moth Plodia interpunctella against a virus (Boots and Begon, 1993), in the mosquito Aedes aegypti against the malaria pathogen (Yan et al., 1997) and in the snail Biomphalaria glabrata against a schistosome (Webster and Woolhouse, 1999). These studies have found that the evolution of improved resistance tends to be associated with reduced development time, size or fecundity, factors that are likely to be positively correlated with fitness. However, currently the most popular technique to investigate costs, artificial selection, is not without problems (Reznick, 1985; Fry, 2003). For example, the type and strength of selection imposed in the laboratory may not be representative of that experienced by the organism in its evolutionary history. It is also important to work with sufficiently large effective population sizes to avoid issues associated with linkage disequilibrium and the fixation of initially rare deleterious mutations that might be mistaken for correlated costly responses. Finally, the technique requires that sufficient additive genetic variation for resistance is segregating in the experimental population at the time of the study.

$D$. melanogaster is attacked by several parasitoid species (Hymenopteran wasps) that lay their eggs into early instar fly larvae. The host attempts to kill the parasitoid egg by means of encapsulation, a chiefly cellular immune response in which haemocytes circulating in the haemolymph recognize the foreign body and form a capsule that melanises leading to the death of the parasitoid egg (Nappi, 1981; Eslin and Prévost, 2000; Lanot et al., 2001; Lavine and Strand, 2002; Meister and Lagueux, 2003). Flies that are artificially selected for increased resistance to parasitoids are poorer larval competitors suggesting a cost to greater investment in 
defence (Kraaijeveld and Godfray, 1997; Fellowes et al., 1998). Selected lines have increased densities of circulating haemocytes that may aid capsule formation, but ingest food more slowly, which may be responsible for their reduced competitive ability (Fellowes et al., 1999; Kraaijeveld et al., 2001). The trade-off may reflect a switch in investment from trophic to defensive functions, or possibly the transport properties of the haemolymph are altered by the increased cell density. There are considerable within- and between-population variations in the ability to encapsulate parasitoid eggs (Carton and Bouletreau, 1985; Kraaijeveld and van Alphen, 1995) and this might be due to a combination of costs to resistance and fluctuating spatial and temporal risks of parasitism.

While defence against macroparasites is chiefly cellular, the main defences against microparasites appear to be largely humoral, with haemocytes playing a more minor role (for example, involving phagocytosing fungi and releasing compounds involved in the activation of the humoral response; Agaisse et al., 2003; Irving et al., 2005). Conserved motifs on the surface of bacteria and fungi are recognized by specialized proteins that trigger one or more signalling cascades resulting in the production of antimicrobial peptides (Lemaitre et al., 1997; Tzou et al., 2002; Hoffmann, 2003; Wang and Ligoxygakis, 2006). Some peptides are upregulated in response to a wide range of microbial challenges, whereas others are more specific, for example, drosomycin and metchnikowin are synthesized in response to fungal attack (Lemaitre et al., 1997; Hultmark, 2003). Research on antimicrobial peptides and other humoral defences has focused chiefly on physiology and genetics rather than ecology and evolution; we know little about the frequency at which flies are subjected to microbial infection in the field and whether investment in these defences is costly. There is, however, some evidence for genetic variation in resistance to microbial pathogens in Drosophila. Lazarro et al. (2004) reported withinpopulation variation in resistance to the entomopathogenic bacterium Serratia marcescens in a North American population of D. melanogaster and Tinsley et al. (2006) showed both between- and within-population variability in susceptibility to the fungus $B$. bassiana.

We are not aware of any studies on the costs of resistance to fungal pathogens in animals (although costs of fungal resistance have been identified in plants; Biere and Antonovics, 1996), and genetic variation for resistance to fungi in insects is seldom reported. Nielsen et al. (2005) could detect no genetic variation among Lymantria dispar strains in susceptibility to the fungal pathogen Entomophaga maimaiga and Blanford et al. (2005) reported no cases on the evolution of resistance to fungal biopesticides. Variation does exist among pea aphid (Acyrtosiphon pisum) clones in resistance to the fungal pathogen Pandora (Erynia) neoaphidis (Ferrari et al., 2001), although this may be due to differences in the secondary endosymbionts they carry (Scarborough et al., 2005, Ferrari et al., 2007). It is unclear, although, whether this lack of evidence of genetic variation for fungal resistance in insects is real or due to the lack of study.

$B$. bassiana is a common soil-borne entomopathogenic fungus, infecting a broad range of insects (Goettel, 1992). The infection process begins when a spore comes in contact with the insect cuticle. It then germinates, penetrates the cuticle and starts to develop hyphae within the insect body, killing it in the process. The insect cadaver then mycoses, producing the next generation of spores (Hajek and St Leger, 1994; Gillespie et al., 2000). To our knowledge, there are no specific reports in the literature of B. bassiana infecting Drosophila in nature, and we use this species as a representative polyphagous fungal pathogen.

In this paper, we report an artificial selection experiment designed to explore the evolution of resistance to a fungal pathogen, B. bassiana, in D. melanogaster. We ask whether there is sufficient additive genetic variation in this trait for increased resistance to evolve, and, if so, whether there are correlated responses that might represent a cost to defence. We use the same large, outbred fly population as in our previous experiments exploring resistance against parasitoids. These showed considerable genetic variation for resistance against two different parasitoid species (Kraaijeveld and Godfray, 1997; Fellowes et al., 1998) and for competitive ability (Sanders et al., 2005). The fungus attacks the adult fly, and we were interested in both the ability of the fly to evolve resistance to infection by the fungus and evolve greater tolerance to the effects of infection.

\section{Materials and methods}

\section{Flies and fungus}

The D. melanogaster population we used was established from flies collected in 1994 near Leiden, The Netherlands, and kept since then in the laboratory in four large, outbred sub-populations. The four sub-populations were merged into one base population, which was reared for two generations before the start of the selection experiment. Flies were cultured in bottles on yeast/sugar/ Kalmus medium supplemented with live baker's yeast. All fly culturing and experiments were done at $20 \pm 1^{\circ} \mathrm{C}$, 16:8 light/dark.

B. bassiana strain 80.2 (Lemaitre et al., 1997) was stored as a stock spore suspension $\left(10^{7}\right.$ spores per $\left.\mathrm{ml}\right)$ in $25 \%$ glycerol at $-80{ }^{\circ} \mathrm{C}$. Cultures were generated from this stock by spreading $5 \mu \mathrm{l}$ of the solution onto Petri dishes with Sabouraud dextrose agar (SDA) and culturing for 3-4 weeks at $29 \pm 0.5^{\circ} \mathrm{C}$. These plates were then used to culture the next set of Sabouraud dextrose agar plates (inoculated by streaking with a nichrome wire loop), which when mature (after 3-4 weeks) were used in the experiments. Before use, samples from all plates were examined under a microscope $(\times 40$ magnification with phase three light) to make sure the fungus colonies were mature and sporulating.

\section{Selection protocol}

The base population was split into five sub-populations, from which five pairs of control and selection lines were initiated. At the beginning of the artificial selection procedure, we let flies from each sub-population lay eggs and collected 1000 of the resulting pupae. These pupae were kept in four bottles with medium, live yeast and a piece of paper towel until the flies emerged. Flies from two randomly chosen bottles, destined to become the selection line of the pair, were anaesthetized briefly and transferred to two plates containing sporulating fungus colonies. The plates were shaken gently for $10-15 \mathrm{~s}$ and then placed, with the lid closed, inside a $30 \times 30 \times 30 \mathrm{~cm}$ 
Perspex cage with a muslin sleeve. Upon recovering from the $\mathrm{CO}_{2}$ anaesthesia, flies picked up more spores by walking on the fungus colony. After being enclosed in the dishes for $3 \mathrm{~h}$, the flies were released into the cage. Flies from the remaining two bottles (destined to become the control line of the pair) were treated similarly, except that the plates contained filter paper rather than a fungus colony. A Petri dish containing medium and live yeast was placed in each cage to allow the flies to feed and oviposit. Each day this was exchanged for a fresh Petri dish, and the original dish was retained. The number of dead flies in the selection- and control-line cages was counted and the cadavers removed. In the control cage, we removed additional flies (without regard to their sex) so that the total number of flies removed from each paired control and selection cage was the same. Larvae in the Petri dishes that had been removed from the cages and retained were allowed to pupate. To initiate the next generation of each selection line, we took 500 pupae from the two most recent Petri dishes that together contained 500 pupae. These were the progeny of relatively old flies, the logic behind this being that it would impose the strongest selection for fungal resistance. Control lines were initiated from pupae collected from Petri dishes of exactly the same age as those used for the selection lines. The experiment was continued for 15 generations.

\section{Survival}

We performed survival assays after 5, 10 and 15 generations of selection. To avoid maternal effects, we first reared flies for two generations without infection and with ad libitum food. Flies were then allowed to oviposit, and when the larvae had pupated, two batches of 150 pupae from each line were collected and kept in bottles, as described above, until the flies emerged. To control for possible variation in spore production and virulence among plates, we divided five plates with sporulating fungi into two separate semicircular arenas using a piece of acetate sheet. One arena of each plate was used for infecting control flies and the other arena for infecting selection flies. One of the two batches of flies from each cage was infected and the other sham infected, in the same way as in the main selection procedure. Flies were then kept in $20 \times 20 \times 20 \mathrm{~cm}$ Perspex cages with a muslin sleeve. Honey and water were provided and the number of flies that died each day was counted and the cadavers removed. We terminated the experiment after 40 days and counted any flies still alive.

Data were analyzed using statistical survival analysis. We assumed a Weibull distribution, which was fitted to the data on longevity with the flies still alive at the end of the experiment treated as censored data. The average time to death for each cage was estimated as $\alpha \Gamma(1 / \gamma+1)$, where $\alpha$ and $\gamma$ are the Weibull distribution's scale and shape parameters, respectively (Fytrou et al., 2006).

\section{Fecundity}

Differences in fecundity across lines were assessed once, at the end of the experiment after 15 generations of selection. As before, to avoid any maternal effects, we first reared flies for two generations without infection. Then, for each line, we let flies lay eggs and their offspring develop, and collected two batches of 100 pupae. When flies emerged, we used the same divided arenas as described above for the survival assay to infect flies from paired control and selection lines. After infection, flies were released into $30 \times 30 \times 30 \mathrm{~cm}$ Perspex cages with a muslin sleeve. Everyday we counted and removed all dead flies in the cages and placed a large $(15 \mathrm{~cm}$ diameter) Petri dish with medium and live yeast in the cage on which flies were allowed to oviposit. Preliminary experiments had shown that a dish of this size contained enough food to adequately feed the total daily offspring production of flies within a cage. We maintained each of the five groups of the four cages (that is, infected and uninfected flies from one pair of control and selection flies) until all infected flies in that group had died. At that point, we counted the number of uninfected flies still alive. We kept the dishes until all larvae had pupated and then froze and stored them at $-20^{\circ} \mathrm{C}$ until the pupae could be counted.

\section{Results}

\section{Survival}

Adult fly survival (Figure 1) was strongly affected by fungal infection $\left(\mathrm{F}_{3,14}=172.5, P<10^{-6}\right)$ but there was no effect of selection history $\left(F_{3,14}=1.41, P=0.28\right)$ or an interaction between infection status and history $\left(\mathrm{F}_{1,14}=2.33, \quad P=0.12\right.$; repeated-measures analysis of variance). Flies from paired control and selection lines were infected with fungus using the same spore plate, and it is possible that these might vary in the density or virulence of spores, and that this variation might obscure a difference between control and selection lines. To exclude this, we performed paired $t$-tests on the time to death of infected flies from control and selection lines after 5, 10 and 15 generations (the tests were one-sided, as we have a clear $a$ priori prediction about the direction of any difference). No significant difference was found (5 generations: $t_{4}=1.24, P=0.14 ; 10$ generations: $t_{4}=0.80, P=0.24 ; 15$ generations: $t_{4}=1.79, P=0.07$ ). Although this last value is near significant in the expected direction, this trend was not consistent across the three assays (Figure 1).

\section{Fecundity}

Where fluctuations in population size can be ignored, fitness equals lifetime reproductive success $\left(w=\sum_{x} l_{x} m_{x}\right)$,

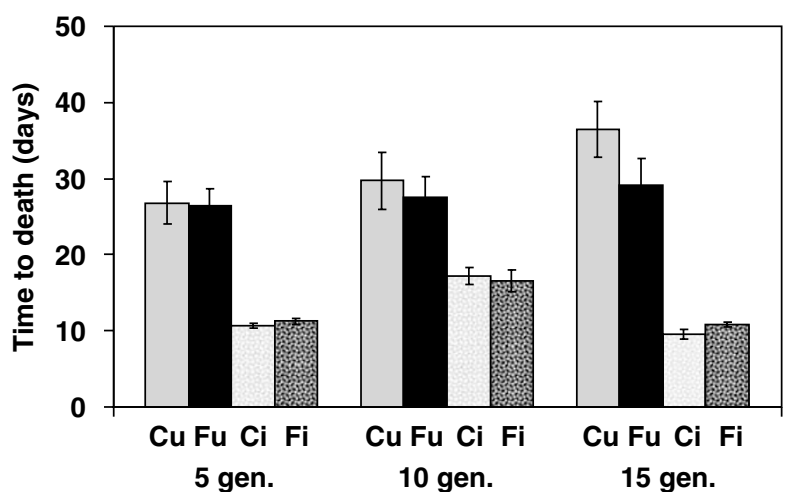

Figure 1 Average time to death of control (C) and selected (F) flies when infected (i) or uninfected $(u)$ by the fungal pathogen. The survival assays were performed after 5, 10 and 15 generations of selection. 
which is the sum of the products of fecundity at age $x\left(m_{x}\right)$ and the probability of living to that age $\left(l_{x}\right)$. Figure $2 \mathrm{a}$ shows the total reproductive success for control and selected flies when infected or uninfected by the fungus and in Figure $2 \mathrm{~b}$ this is broken down into the age-specific contributions to fitness.

Selection for fungal resistance does not seem to have increased lifetime reproductive success in the presence of the fungal pathogen (one-sided $t$-test: $t_{8}=0.30, P=0.39$; a paired $t$-test that controls for differences in spore plates gives a similar result). However, in the absence of fungal infection, flies selected for improved performance against the fungus have significantly lower lifetime reproductive success compared with the controls (one-sided $t$-test: $\left.t_{8}=2.03, P=0.038\right)$. In Discussion, we explore whether this might result from a trade-off between an aspect of resistance and some other component of fitness, or if it might be an accidental by-product of the selection protocol.

Surprised by the lack of response to selection, we examined the age-specific fitness contributions for possible more subtle responses to selection. Figure $2 b$ suggests that selection flies have greater late-life fitness than control flies, which may indicate a better ability by older flies to cope with fungal infection (recall that the next generation is initiated from the eggs of older flies). We tested this by comparing the day at which each fly achieved $50 \%$ of its total reproductive success (Figure 3 ). Infected flies from selection lines passed this barrier at a significantly older age than those from control lines ( $t$-test: $t_{8}=2.53, P=0.036$; as we were concerned about the applicability of the $t$-statistic, we also did a permutation test in which the observed difference was greater than the 97.5 percentile of 10000 permutations).

There was no difference in the same statistic measured on uninfected control and selection flies $\left(t_{8}=0.61\right.$, $P=0.28$ ). Note that these are tests of an a posteriori hypothesis and the quoted probability values should be treated as informal tests of significance. In this experiment, we found no difference in female life span between control and selected lines $\left(F_{1,16}=0.15, \quad P=0.71\right.$; Figure 4a). Thus, the difference in late-life fitness is more likely due to differences in $m_{x}$ than $l_{x}$. This is confirmed by Figure $4 b$, where we have plotted the fecundity of flies that are still alive $\left(m_{x}\right)$ against time, which gives a pattern very similar to that of total fitness in Figure $2 b$.

\section{Discussion}

We draw three main conclusions from this experiment, which we discuss in turn. First, a strong response to selection for increased resistance to fungal pathogens is not seen, as has been found in earlier experiments in which Drosophila has been selected to counter natural enemies. Second, we have suggestive evidence that Drosophila can evolve greater late-life fitness in the presence of fungal infection. Third, flies selected for resistance to the fungus have lower fitness in the absence of the infection, which might be evidence of evolutionary trade-off.

D. melanogaster rapidly evolves resistance to some of its main larval parasitoids, the fraction of individuals surviving increasing from less than 5\% to around $45 \%$ (Kraaijeveld and Godfray, 1997; Fellowes et al., 1998). The precise mechanisms involved are not fully understood but probably involve an increase in the density of haemocytes, cells that circulate in the body cavity (haemocoel) and are involved in encapsulation. The

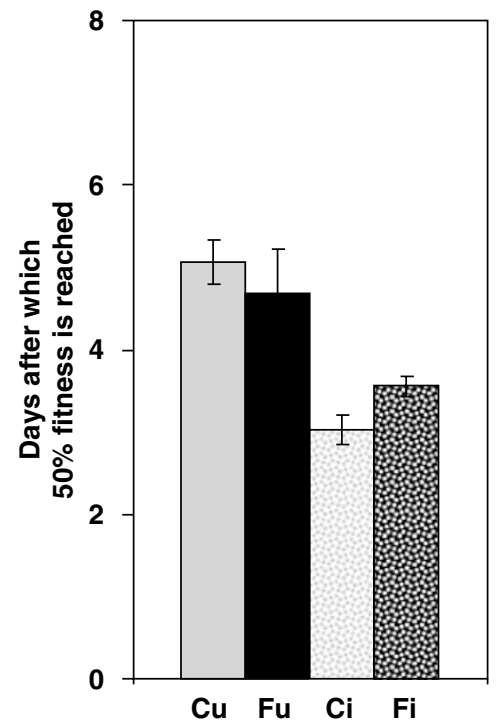

Figure 3 The day on which $50 \%$ of total lifetime reproductive success was achieved by control (C) and selected (F) flies when infected (i) or uninfected (u) by the fungal pathogen.
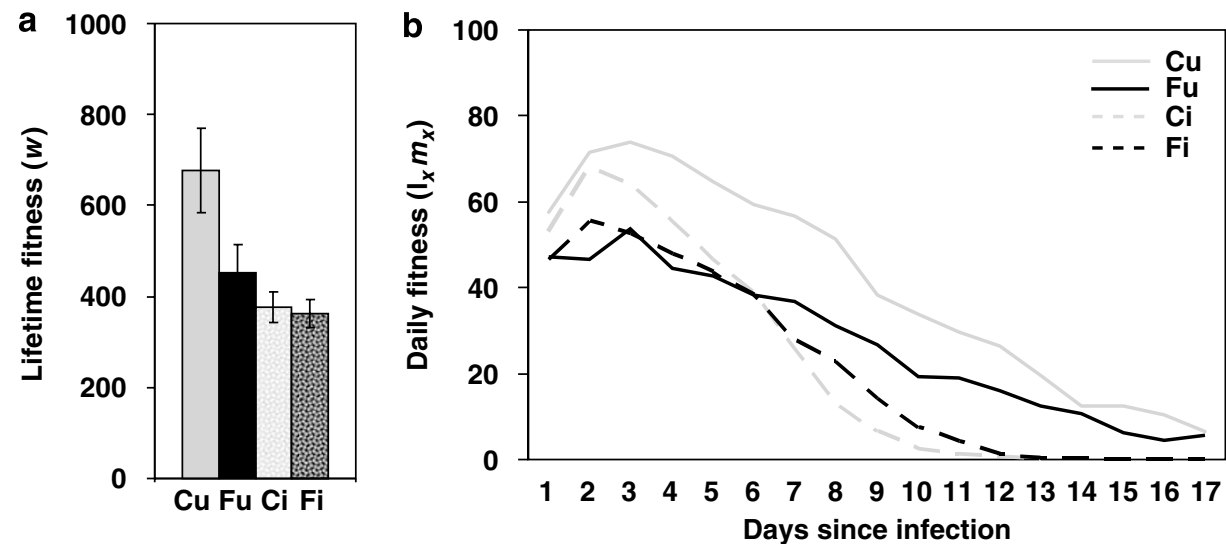

Figure 2 Overall lifetime reproductive success (a) and the contribution to lifetime reproductive success of flies of different ages (b) of control $(\mathrm{C})$ and selected $(\mathrm{F})$ flies when infected (i) or uninfected $(\mathrm{u})$ by the fungal pathogen. 

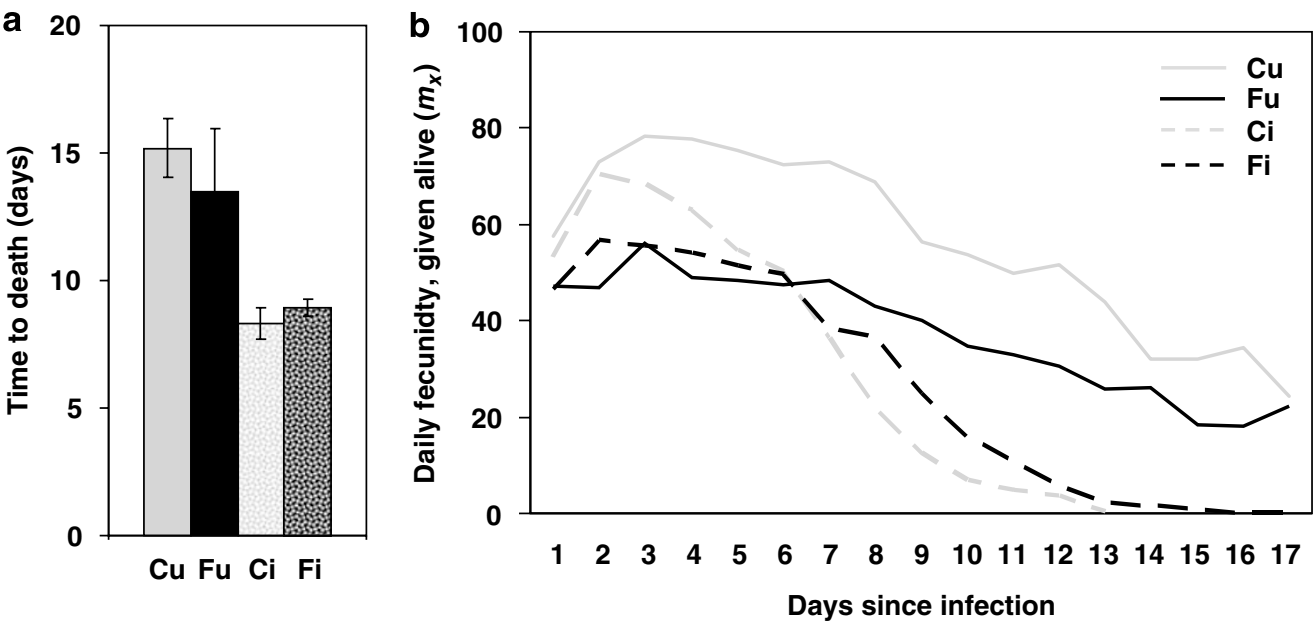

Figure 4 Average time to death of female control (C) and selected (F) flies (a) and daily fecundity of control (C) and selected (F) flies (b) when infected (i) or uninfected (u) by the fungal pathogen.

changes in survival or fecundity we detected in the current experiments were much more subtle and need further confirmation. There are several possible reasons for this difference in response. First, defence against microbial pathogens tends to involve the humoral rather than the cellular arm of the invertebrate immune system, and possibly there is less additive genetic variation segregating in populations for this trait. This, in turn, might be an intrinsic property of the humoral defence system, reflect less variability in the selection pressure exerted by microbial compared to larger pathogens or parasites or be due to fewer trade-offs between investment in humoral defence and other fitness components compared with investment in cellular defence. Alternatively, microbial pathogens may be less important in the ecology of $D$. melanogaster compared with parasitoids and larger pathogens. Evaluating this latter hypothesis is difficult because despite its importance in genetics and other fields, the ecology of this species in the field is still relatively poorly known. Third, a major difference between parasitoids and fungal pathogens is that the outcome of parasitoid attack is essentially binary; the fly larva dies or becomes an adult whose fitness is only slightly reduced because of parasitism, whereas the effect of fungal infection as an adult is less severe and more graded. Finally, it is possible that the spore dose used in our experiments was higher than that typically received by flies in the field and that this prevented the evolution of resistance. Unfortunately, we know little about the typical exposure of Drosophila to fungal pathogens in nature. In another experiment involving the same host and pathogen, Tinsley et al. (2006) found considerable between- and within-population variations in time to death of $D$. melanogaster after infection with B. bassiana. In their experiments, flies typically took longer to die after infection than in our experiments but as their infection protocol differs in several ways from ours, it is difficult to compare the spore doses the flies received. A further issue is that we fed flies honey in the survival and fecundity experiments. Honey is known to have antimicrobial activity (Wahdan, 1998) and it is possible that ingesting antimicrobial compounds somehow augments a fly's antifungal resistance. However, there is no indication that selection and control flies, or infected and uninfected flies, consumed different amounts of honey.

Post facto study of the mortality and fecundity schedules suggests that compared with control lines selected lines produce more offspring late in life when infected with fungal pathogens. This result needs confirmation from new experiments but is consistent with the form of selection we imposed on the lines. We deliberately initiated new generations using the eggs of older flies to maximize selection on increased survival from, and tolerance of, fungal infection. We were aware that this might select for investment in late-life reproduction and so carefully initiated new generations of control lines with flies of exactly the same age as the selection lines. Increased reproduction late in life in the presence of infection (see comparison of infected selection and control lines in Figure 2) was thus an expected response to our selection pressure. However, we had assumed that overall lifetime reproductive success in the presence of infection would also be higher in the selection lines. This did not occur because the early-life contribution was lower in the selection lines. Because we used only relatively old flies to initiate the new generations, this drop in early-life reproduction would not have had fitness consequences in the environment under which the flies were selected. Indeed, the drop in early-life reproduction might be a reflection of the well-established trade-off between early- and late-life fitness. Note that the relatively higher late-life reproductive success was only found in comparisons of infected and not uninfected flies. This suggests that the fly has evolved a facultative rather than constitutive response to infection.

Our third conclusion is that selected flies have reduced fitness in the absence of fungal infection compared with control flies. This cannot be explained by differential inbreeding effects, as care was taken to keep population sizes of control and selection lines equal at all times. If the changes in late-life reproduction in the presence of fungal infection are real, then possibly the reduction in fitness reflects a trade-off between pathogen resistance or tolerance and some other aspect of fitness. An alternative is that the selection we have imposed is so strong that one or more deleterious mutations have hitchhiked to fixation. Given the likely strength of selection in the 
experiments, and the population size in the selection and control lines, we assume that this is a less likely explanation. Finally, if the fungal infection is vertically transmitted, it might explain the reduced fitness of infected lines. However, we have never observed fungal infections arising except through infection of the adult, and are aware of no evidence of Beauvaria causing latent infections in any of its hosts.

We do not know the mechanistic basis of the differences between the control and selection lines, for example, whether they involve differences in the production of cecropin, drosomycin or metchnikowin, the antimicrobial peptides that target fungi in D. melanogaster (Hultmark, 2003). However, as selected flies do not live longer after infection than control flies, we may not have selected for resistance in the typical sense. Organisms can combat natural enemies either by attacking them directly (that is, resistance) or by tolerance, reducing their detrimental effects (Roy and Kirchner, 2000). Tolerance is a particularly common strategy for plants attacked by herbivores and pathogens (van der Meijden et al., 1988; Simms and Triplett, 1994; Strauss and Agrawal, 1999). The ability of selected flies in our experiments to produce more eggs late in life may be an example of increased tolerance to a pathogen, although further physiological studies are needed to explore this idea.

Fungal pathogens are used as biopesticides, for example against locusts, while new applications such as combating the mosquito vectors of malaria are being explored (Kooyman et al., 1997; Lomer et al., 2001; Blanford et al., 2005; Scholte et al., 2005). Evolution of resistance against pesticides is a continuing problem in pest control. If our results are considered as general representative of the interaction between insects and fungal pathogens, they might suggest that resistance to fungal biopesticides will not evolve easily.

\section{Acknowledgements}

We thank Marie Meister (CNRS, IBMC, Strasbourg) for providing the fungus and Meirion Hopkins and Gary Needham for offering invaluable assistance with the selection procedure and subsequent assays.

\section{References}

Agaisse H, Petersen U-M, Boutros M, Mathey-Prevot B, Perrimon N (2003). Signaling role of hemocytes in Drosophila JAK/STAT-dependent response to septic injury. Dev Cell 5: 441-450.

Biere A, Antonovics J (1996). Sex-specific costs of resistance to the fungal pathogen Ustilago violacea (Microbotryum violaceum) in Silene alba. Evolution 50: 1098-1110.

Blanford A, Chan BHK, Jenkins N, Sim D, Turner RJ, Read AF et al. (2005). Fungal pathogen reduces potential for malaria transmission. Science 308: 1639-1641.

Boots M, Begon M (1993). Trade-offs with resistance to a granulosis virus in the Indian meal moth, examined by a laboratory evolution experiment. Funct Ecol 7: 528-534.

Carton Y, Bouletreau M (1985). Encapsulation ability of Drosophila melanogaster: a genetic analysis. Dev Comp Immunol 9: 211-219.

Eslin P, Prévost G (2000). Racing against host's immunity defenses: a likely strategy for passive evasion of encapsulation in Asobara tabida parasitoids. J Insect Physiol 46: $1161-1167$
Fellowes MDE, Kraaijeveld AR, Godfray HCJ (1998). Trade-off associated with selection for increased ability to resist parasitoid attack in Drosophila melanogaster. Proc R Soc London B 265: 1553-1558.

Fellowes MDE, Kraaijeveld AR, Godfray HCJ (1999). Association between feeding rate and parasitoid resistance in Drosophila melanogaster. Evolution 53: 1302-1305.

Ferrari J, Müller CB, Kraaijeveld AR, Godfray HCJ (2001). Clonal variation and covariation in aphid resistance to parasitoids and a pathogen. Evolution 55: 1805-1814.

Ferrari J, Scarborough CL, Godfray HCJ (2007). Genetic variation in the effect of a facultative symbiont on host plant use by pea aphids. Oecologia 153: 323-329.

Fry JD (2003). Detecting ecological trade-offs using selection experiments. Ecology 84: 1672-1678.

Fytrou A, Schofield PG, Kraaijeveld AR, Hubbard SF (2006). Wolbachia infection suppresses both host defence and parasitoid counter-defence. Proc R Soc London B 273: 791-796.

Gillespie JP, Bailey AM, Cobb B, Vilcinskas A (2000). Fungi as elicitors of insect immune responses. Arch Insect Biochem Physiol 44: 49-68.

Goettel MS (1992). Fungal agents for biocontrol. In: Lomer CJ, Prior C (eds). Biological Control of Locusts and Grasshoppers. CABI: Wallingford, pp 122-132.

Hajek AE, St Leger RJ (1994). Interactions between fungal pathogens ands insect hosts. Annu Rev Entomol 39: 293-322.

Hoffmann JA (2003). The immune response of Drosophila. Nature 426: 33-38.

Hultmark D (2003). Drosophila immunity: paths and patterns. Curr Opin Immunol 15: 12-19.

Irving $\mathrm{P}$, Ubeda J-M, Doucet $\mathrm{D}$, Troxler L, Lagueux $\mathrm{M}$, Zachary D et al. (2005). New insights into Drosophila larval hemocyte functions through genome-wide analysis. Cell Microbiol 7: 335-350.

Kooyman C, Bateman RP, Langewald J, Lomer CJ, Ouambama Z, Thomas MB (1997). Operational-scale application of entomopathogenic fungi for control of Sahelian grasshoppers. Proc $R$ Soc London B 264: 541-546.

Kraaijeveld AR, Godfray HCJ (1997). Trade-off between parasitoid resistance and larval competitive ability in Drosophila melanogaster. Nature 389: 278-280.

Kraaijeveld AR, Limentani EC, Godfray HCJ (2001). Basis of the trade-off between parasitoid resistance and larval competitive ability in Drosophila melanogaster. Proc R Soc London B $\mathbf{2 6 8}$ 259-261.

Kraaijeveld AR, van Alphen JJM (1995). Geographic variation in encapsulation ability of Drosophila melanogaster larvae and evidence for parasitoid-specific components. Evol Ecol 9: 10-17.

Lanot R, Zachary D, Holder F, Meister M (2001). Postembryonic hematopoiesis in Drosophila. Dev Biol 230: 243-257.

Lavine MD, Strand MR (2002). Insect hemocytes and their role in immunity. Insect Biochem Mol Biol 32: 1295-1309.

Lazarro BP, Sceurman BK, Clark AG (2004). Genetic basis of natural variation in $D$. melanogaster antibacterial immunity. Science 303: 1873-1876.

Lemaitre B, Reichhart J-M, Hoffmann JA (1997). Drosophila host defense: differential induction of antimicrobial peptide genes after infection by various classes of microorganisms. Proc Natl Acad Sci USA 94: 14614-14619.

Lomer CJ, Bateman RP, Johnson DL, Langewald J, Thomas MB (2001). Biological control of locusts and grasshoppers. Annu Rev Entomol 46: 667-702.

Meister M, Lagueux M (2003). Drosophila blood cells. Cell Microbiol 5: 573-580.

Nappi AJ (1981). Cellular immune response of Drosophila melanogaster against Asobara tabida. Parasitology 83: 319-324.

Nielsen C, Keena M, Hajek AE (2005). Virulence and fitness of the fungal pathogen Entomophaga maimaiga in its host, Lymantria dispar, for pathogen and host strains originating from Asia, Europe, and North America. J Invert Pathol 89: 232-242. 
Reznick D (1985). Cost of reproduction: an evaluation of the empirical evidence. Oikos 44: 257-267.

Rolff J, Siva-Jothy MT (2003). Invertebrate ecological immunity. Science 301: 472-475.

Roy BA, Kirchner JW (2000). Evolutionary dynamics of pathogen resistance and tolerance. Evolution 54: 51-63.

Sanders AE, Scarborough C, Layen SJ, Kraaijeveld AR, Godfray HCJ (2005). Evolutionary change in parasitoid resistance under crowded conditions in Drosophila melanogaster. Evolution 59: 1292-1299.

Scarborough CL, Ferrari J, Godfray HCJ (2005). Aphid protected from pathogen by endosymbiont. Science 310: 1781.

Schmid-Hempel P, Ebert D (2003). On the evolutionary ecology of specific immune defence. Trends Ecol Evol 18: 27-32.

Scholte E-J, Ng'habi K, Kihonda J, Takken W, Paaijmans K, Abdulla $S$ et al. (2005). An entomopathogenic fungus for control of adult African malaria mosquitoes. Science 308: 1641-1642.

Sheldon BC, Verhulst SE (1996). Ecological immunology: costly parasite defences and trade-offs in evolutionary ecology. Trends Ecol Evol 11: 317-321.

Simms EL, Triplett J (1994). Costs and benefits of plant responses to disease: resistance and tolerance. Evolution 48 : 1933-1945.
Strauss SY, Agrawal AA (1999). The ecology and evolution of plant tolerance to herbivory. Trends Ecol Evol 14: 179-185.

Tinsley MC, Blanford S, Jiggins FM (2006). Genetic variation in Drosophila melanogaster pathogen susceptibility. Parasitology 132: 767-773.

Tzou P, De Gregorio E, Lemaitre B (2002). How Drosophila combats microbial infection: a model to study innate immunity and host-pathogen interactions. Curr Opin Microbiol 5: 102-110.

van der Meijden E, Wijn M, Verkaar HJ (1988). Defense and regrowth, alternative plant strategies in the struggle against herbivores. Oikos 51: 355-363.

Wahdan HA (1998). Causes of the antimicrobial activity of honey. Infection 26: 26-31.

Wang L, Ligoxygakis P (2006). Pathogen recognition and signalling in the Drosophila innate immune response. Immunobiology 211: 251-261.

Webster JP, Woolhouse MEJ (1999). Cost of resistance: relationship between reduced fertility and increased resistance in a snail-schistosome host-parasite system. Proc $R$ Soc London $B$ 266: 391-396.

Yan G, Severson DW, Christensen BM (1997). Costs and benefits of mosquito refractoriness to malaria parasites: implications for genetic variability of mosquitoes and genetic control of malaria. Evolution 51: 441-450. 